\title{
Implementation of a Blowing Boundary Condition in the LAURA Code
}

\author{
Richard A. Thompson ${ }^{1}$ and Peter A. Gnoffo ${ }^{2}$ \\ NASA Langley Research Center, Hampton, Virginia
}

\begin{abstract}
Preliminary steps toward modeling a coupled ablation problem using a finite-volume Navier-Stokes code (LAURA) are presented in this paper. Implementation of a surface boundary condition with mass transfer (blowing) is described followed by verification and validation through comparisons with analytic results and experimental data. Application of the code to a carbon-nosetip ablation problem is demonstrated and the results are compared with previously published data. It is concluded that the code and coupled procedure are suitable to support further ablation analyses and studies.
\end{abstract}

\section{Nomenclature}

$B=$ blowing parameter

$C=$ species mass fraction

$f=$ Blasius function

$h=$ static enthalpy

$\mathrm{h}^{*} \quad=$ heat transfer coefficient

$H \quad=$ total enthalpy

$H_{r} \quad=$ recovery enthalpy

$L \quad=$ reference length

$\mathrm{m} \quad=$ mass flow rate

$p \quad=$ pressure

$q \quad=$ heat transfer rate

Re $\quad=$ Reynolds number

$\mathrm{r}_{\mathrm{n}}, \mathrm{R}_{\mathrm{n}} \quad=$ sphere nose radius

$\mathrm{S}=$ surface distance

St $\quad=$ Stanton number

$\mathrm{t}=$ time

$T=$ temperature

$\mathrm{u}, \mathrm{V}=$ streamwise and normal velocity

$\mathrm{x}, \mathrm{y}, \mathrm{z}=$ coordinate directions

$\lambda=$ correction factor

$\eta=$ Blasius coordinate

$\rho \quad=$ density

Subscripts

$i=$ species number

$e \quad=$ boundary layer edge condition

o $\quad=$ stagnation condition

ref $\quad=$ reference condition

$\mathrm{x}=$ streamwise direction

$\mathrm{W} \quad=$ wall condition

$0=$ condition with zero mass transfer

$1=$ condition at volume face one-half cell above wall

$2=$ condition at volume face one-half cell below wall

\footnotetext{
${ }^{1}$ Engineer, Aerothermodynamics Branch, MS 408A, Senior Member AIAA.

${ }^{2}$ Senior Research Engineer, Aerothermodynamics Branch, MS 408A, AIAA Fellow.
} 


\section{Introduction}

$\mathrm{T}$ The Crew Exploration Vehicle (CEV) is being currently designed to support manned missions returning from low Earth orbit and the moon. At lunar return velocities, all currently available thermal protection systems (TPS) will rely on an ablative heatshield design to absorb the high energies associated with Earth reentry. The modeling and design of these heatshields is traditionally done using an uncoupled approach where the heating environment is computed in the absence of ablation and is later modified by a blowing correction to determine the TPS material response. However, some efforts at performing a coupled analysis have been reported (Refs. 1-3) where computational solutions of the flowfield and the ablating material were performed in an iterative fashion. While much success has been achieved using an uncoupled analysis for design, it is of interest to obtain higher fidelity (coupled) analyses in order to better assess the system and quantify design margins. This paper will present steps toward those goals by documenting the implementation of a mass-transfer surface boundary condition (blowing) into a state-of-the-art code (LAURA, Ref. 4) that can be used in future coupled analyses. Similar progress has been reported by Martinelli, et. al. (Ref. 5) for another computational fluid dynamics (CFD) code. Modifications to the LAURA code needed to model an ablating surface are described in this paper followed by verification and validation of the results. While mass injection and ablation processes are often turbulent, only laminar flow conditions are considered in this paper. A process for coupling the LAURA code in a surface ablation and recession analysis will be described and results for a carbon nosetip material will be demonstrated.

\section{Implementation}

The LAURA (Langley Aerothermodynamic Upwind Relaxation Algorithm) code (Ref. 4) uses a finite-volume shock-capturing approach to solve steady viscous and inviscid flow problems. The algorithm incorporates pointimplicit or line implicit relaxation schemes to obtain solutions efficiently on multi-processor and massively parallel computers. The code has been successfully applied to a wide range of hypersonic vehicles and flight conditions during its evolution over the past 15 years. LAURA includes models for perfect gas, equilibrium air, and thermal and chemical nonequilibrium air in addition to models for $\mathrm{CF}_{4}$ and a Mars atmosphere. An important feature of the code is the ability to perform one-dimensional grid adaption in parallel with the solution to resolve high gradients in the boundary layer and across a bow shock. For the applications in this work, the thin-layer form of the NavierStokes equations were solved and an eigenvalue limiter of 0.3 was used in the code. The computational grids in every case were adapted such that the cell Reynolds number at the wall was $\mathrm{O}(1)$ and solution convergence was obtained when changes in surface heating were less than $0.1 \%$ after 5000 iterations.

The boundary condition for modeling gas injection at a solid surface is fairly straightforward and was treated in an explicit fashion in the LAURA code. Figure 1 illustrates a computational cell centered on a wall boundary with flow conditions one-half a cell above the surface (point 2) and one-half cell below (point 1). This lower cell is treated as a a pseudo-cell for the purposes here. At the wall, the mass flow rate $\left(\rho_{w} v_{w}\right)$, temperature $\left(T_{w}\right)$, and species mass fractions $\left(c_{i}\right)$ are assumed to be known from a material response code and by assuming chemical equilibrium at the surface.

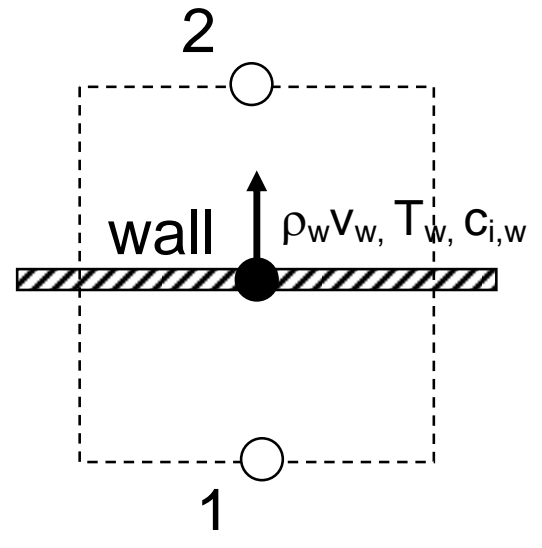

Figure 1. Cell volume for treatment of wall blowing boundary condition 
Assuming no losses through the vertical (side) faces, the conservation of momentum in this cell can be written

$$
p_{2}+\rho_{2} v_{2}^{2}=p_{w}+\rho_{w} v_{w}^{2}
$$

with the massflow at the wall

$$
\dot{m}=\rho_{w} v_{w}
$$

and the pressure and density related by thermodynamics

$$
p_{w}=p_{w}\left(\rho_{w}, T_{w}\right)
$$

This system of three equations in three unknowns can be solved to yield the density, pressure, and velocity at the surface. In this explicit boundary condition treatment, a solution for the surface quantities is performed at each iteration step in LAURA. During development of the boundary condition a simple test was devised using a sphere geometry in Mach 6 air with helium injection at the surface as shown in Figure 2.

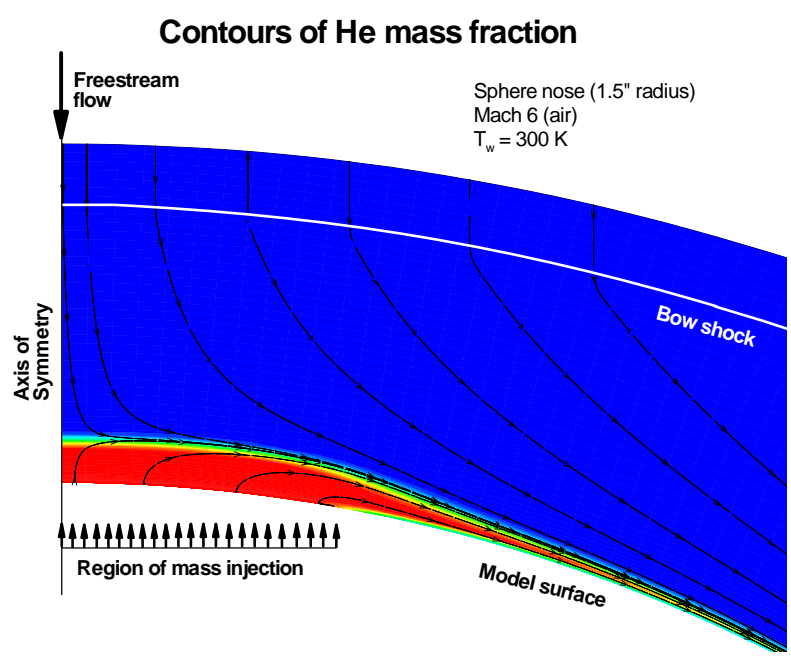

Figure 2. Test case for development of blowing boundary condition

In the test case, a region of mass injection was defined beginning at the stagnation point and ending a short distance downstream. A range of mass flow rates were simulated to test the implementation of the blowing boundary condition and study solution convergence. The figure illustrates the flowfield computed for a case with a moderate blowing rate where the color contours represent the different gases-helium (red) and air (blue). Streamlines from the surface injection and oncoming flow illustrate the computed velocity field. These qualitative results appear as expected and variation of mass flow rates proved the boundary condition scheme to be robust within LAURA for the cases considered.

\section{Results}

The following sections present results that were achieved with the code modifications in order to verify and validate $(\mathrm{V} \& \mathrm{~V})$ the blowing boundary condition implementation. This $\mathrm{V} \& \mathrm{~V}$ process is expected to be a continuous task as new conditions are analyzed and new results are identified that can be used for comparison.

\section{A. Verification}

Initial verification of the blowing boundary condition in LAURA was performed by using the code to compute the theoretical Blasius velocity profile for flow over a flate plate with injection. In the Blasius approximation, the equation for flow over a flate plate is given by

$$
f^{\prime \prime}+f f^{\prime \prime}=0
$$


where the boundary condition for mass flow through the surface, in terms of the Blasius function, is

$$
\frac{\rho_{w} v_{w}}{\rho_{\infty} v_{\infty}}=-\frac{f(0)}{2 \sqrt{\operatorname{Re}_{x}}}
$$

A rectangular computational domain was constructed to compute the Blasius flat plate problem with LAURA. The domain extended $10 \mathrm{~m}$ in the vertical direction and $10 \mathrm{~m}$ from either end of a $1 \mathrm{~m}$ plate in the upstream and downstream directions. As constructed, the CFD problem included the effects of flow moving onto and off the flat plate at the leading and trailing edges, respectively. Figure 3(a) illustrates those effects on the velocity profiles near the plate edges $(x=0 \mathrm{~m}$ and $\mathrm{x}=1.0 \mathrm{~m})$ for the case with no blowing through the surface. As shown in the figure, the velocity profiles near the center of the plate $(0.5<\mathrm{x}<0.75 \mathrm{~m})$ collapse to the Blasius profile when plotted in the similarity variables. A similar plot is shown in Figure 3(b) for additional cases where blowing into the boundary layer is introduced at the surface. The velocity profiles in Figure 3(b) correspond to cases where the Blasius function at the surface, $f(0)$, equals $0.0,-0.4$, and -0.8 . Here, the negative values for $f(0)$ denotes flow into the boundary layer (blowing); no solutions for positive values of $f(0)$ (i.e. for suction) were computed. The data used for comparison in Figure 3 were obtained from the tabulation of Blasius profiles reported by Emmons and Leigh (Ref. 6). Good agreement between the LAURA computed profiles and the Blasius data was found.

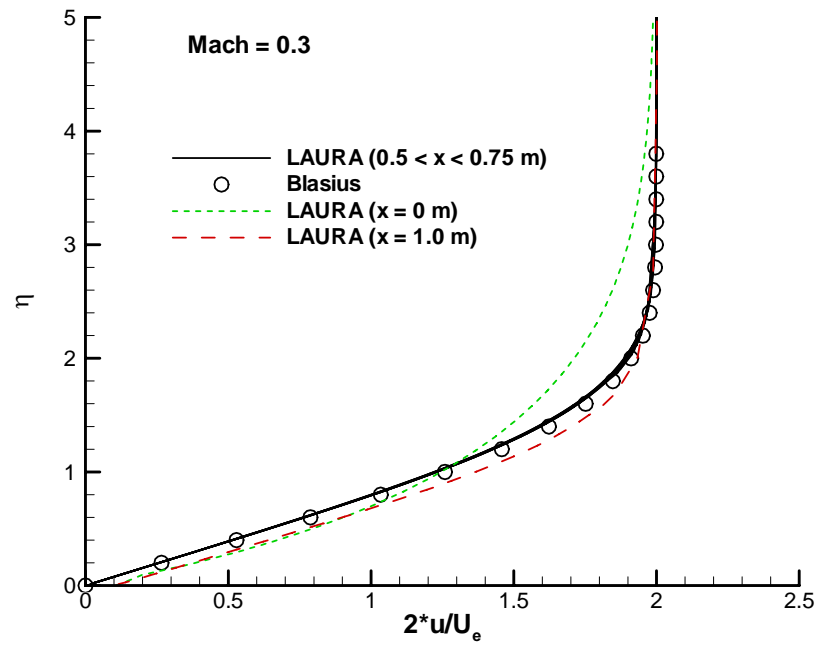

(a) Velocity profiles with no blowing

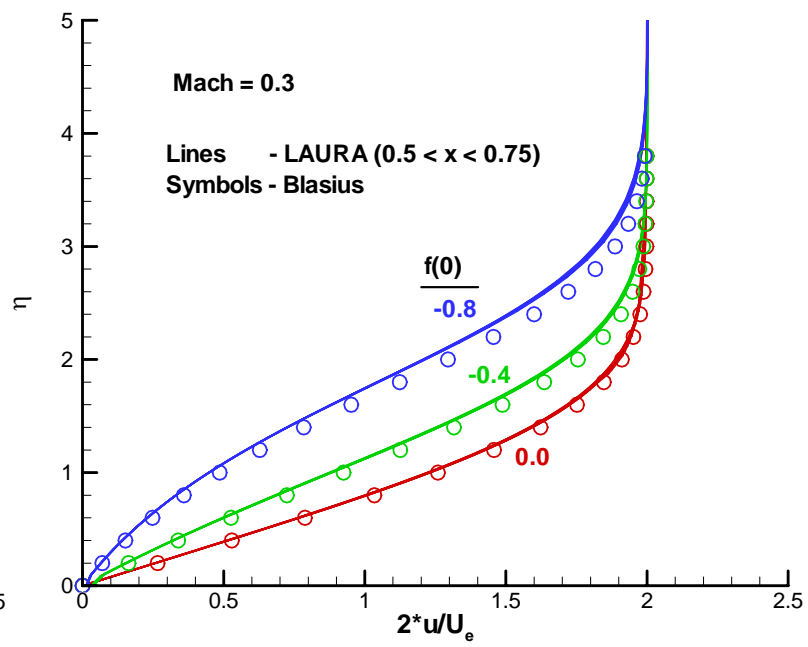

b) Velocity profiles with surface injection

Figure 3. Comparison of predicted velocities with Blasius profiles

An early approach to the analysis of laminar boundary layers with mass transfer was the use of film theory such as reported by Mickley, et.al (Ref. 7). Following the thin-film approximation, it was shown that variation of the transfer coefficients (friction, heating, and diffusion) can be represented by a simple exponential function

$$
\theta=\frac{\phi}{e^{\phi}-1}
$$

where $\theta$ is a ratio of the coefficient with and without mass transfer and $\phi$ is a function of the mass transfer rate. Derivations of the same function have been presented by several authors (Refs. 8-10) and the resulting function for the reduction of heat transfer in the presence of blowing has been widely used. For heat transfer, the function in Eq. 6 is usually written in the form

where the Stanton number is defined by

$$
\begin{gathered}
\frac{S t}{S t_{0}}=\frac{B_{0}}{e^{B_{0}}-1} \\
S t=\frac{q}{\rho_{e} u_{e}\left(H_{r}-h_{w}\right)}
\end{gathered}
$$


and the blowing parameter by

$$
B_{0}=\frac{\rho_{w} v_{w}}{\rho_{e} u_{e} S t_{0}}
$$

The subscript 0 in the preceding equations denotes a quantity taken at a zero mass transfer condition. The assumptions associated with Eq. 4 are that the flow has Couette-like properties so that gradients in the streamwise direction are much smaller than across the layer. In addition, the derivation assumes that the mass flux introduced at the surface is constant across the layer and that the layer thickness is unaffected. There is no explicit requirement (or modeling) for the flow to be laminar or turbulent in the above equations. A slightly modified version of Eq. 4 has the form

$$
\frac{S t}{S t_{0}}=\frac{2 \lambda B_{0}}{e^{2 \lambda B_{0}}-1}
$$

or, equivalently

$$
\frac{S t}{S t_{0}}=\frac{\ln (1+2 \lambda B)}{2 \lambda B}
$$

where

$$
B=\frac{\rho_{w} v_{w}}{\rho_{e} u_{e} S t}
$$

Introduction of the $\lambda$ term in Eq. 10 was reported (Ref. 11) to improve the agreement with turbulent data when $\lambda=0.4$ while reducing to the original formula for laminar flow when $\lambda=0.5$. Equation 11 is the basis for correcting the surface heat transfer in the CMA (Ref. 11) and FIAT (Ref. 12) material response codes when an unblown value of heating $\left(S t_{0}\right)$ is supplied.

To provide further verification of the boundary condition implementation in the LAURA code, a model problem was solved for a Couette flow using the assumptions described above. It was expected that the reduction in surface heat transfer computed with LAURA should match the values predicted by Eqs. 7-9. For the CFD solution, a rectangular domain was constructed with a planar surface representing the wall and freestream conditions prescribed at the outer (edge) location. The upstream and downstream faces of the domain were set to match each other since the flow was assumed constant in the streamwise direction. Perfect gas flow solutions were obtained for a range of edge conditions that included Mach numbers from 0.15 to 3.0, unit Reynolds numbers from $1 \times 10^{4}$ to $1 \times 10^{5} \mathrm{~m}^{-1}$, and wall temperature ratios $\left(T_{w} / T_{o}\right)$ between 0.2 to 0.65 . Results for the computed variation in heat transfer for blowing parameters $(B)$ between 0 and 3 are shown in Figure 4. These computed values from LAURA are compared with the analytic result from Eq. 7 and, while displaying some disparity, clearly show that the level and trend are very closely predicted.

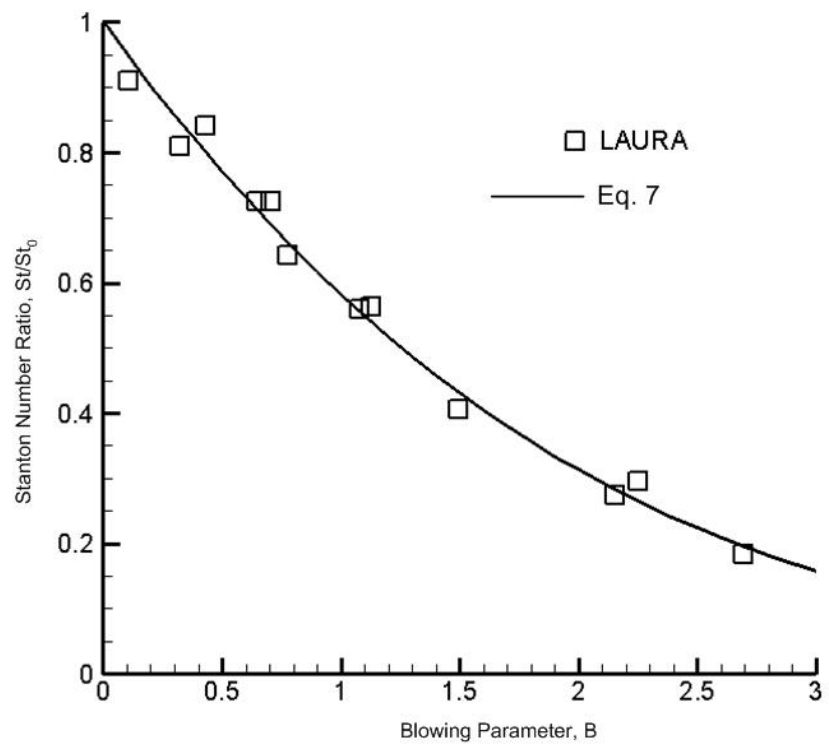

Figure 4. Stanton number reduction as a function of blowing parameter 


\section{B. Validation}

While a substantial amount of theoretical work exists in the literature concerning the effects of blowing on heating rate, there are a limited number of experimental tests reported that can be used for code validation. In the present work, comparisons have been made with the published results of Feldhuhn (Ref. 13), Kaattari (Ref. 14), and Marvin (Ref. 15). Additional sources of data from Libby (Ref. 16) and Laganelli (Ref. 17) were identified during the course of this work but those data were dominated by turbulent flow and comparisons are not presented in this paper.

Feldhuhn measured heat transfer distributions on a porous, spherically blunted cone in Mach 5 air over a substantial range of Reynolds numbers. The 5-deg sphere-cone model was constructed with three interior manifolds such that the mass transfer rates of the injected nitrogen could be varied independently in the stagnation region, the sphere shoulder, and the conical flank. A matrix of tests were performed where the blowing varied between zero and seven times the nominal to yield injection rates up to 2 percent of the freestream massflow. At the higher mass flow rates and Reynolds numbers, the test data clearly showed transition to turbulence at the location of mass injection or downstream on the conical flank. A small number of wind tunnel conditions were tested where the flow was believed to remain laminar over the sphere cone nose and the data from those tests were used for validation in the present work.

Four test conditions were considered for comparison (Cases 15, 22, 23, and 141 in Ref. 13) with each case being at Mach 5 and $50 \mathrm{~atm}$ total pressure which yielded the lowest freestream Reynolds number $\left(3.8 \times 10^{6} \mathrm{ft}^{-1}\right)$. Mass flow in the stagnation region and sphere shoulder was set at zero, once, or twice the nominal value (0.6 percent of freestream). These varying injection rates were defined in LAURA for the CFD computation and a comparison of the measured and predicted heating for these cases is shown in Figure 5(a) and 5(b). The results computed in this work have been cast in terms of heat transfer coefficient and plotted on the same scale and axes as the data was presented in Ref. 13.

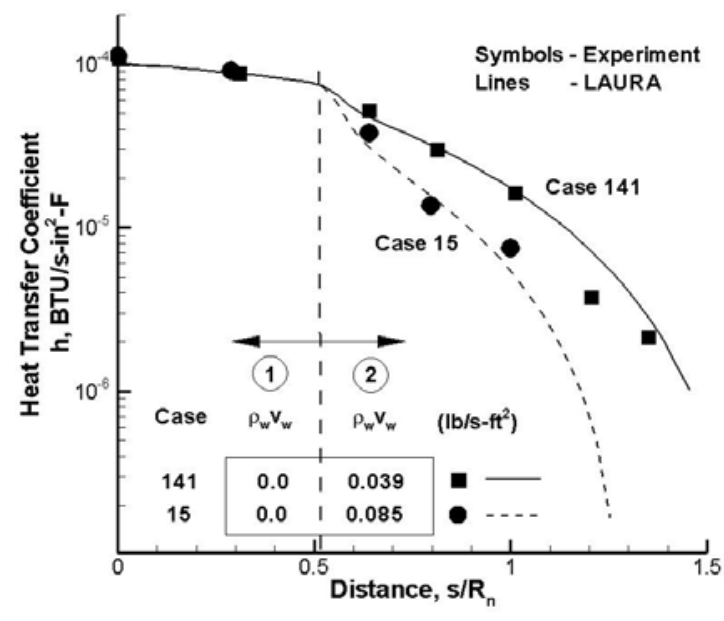

a) Zero blowing in the stagnation region

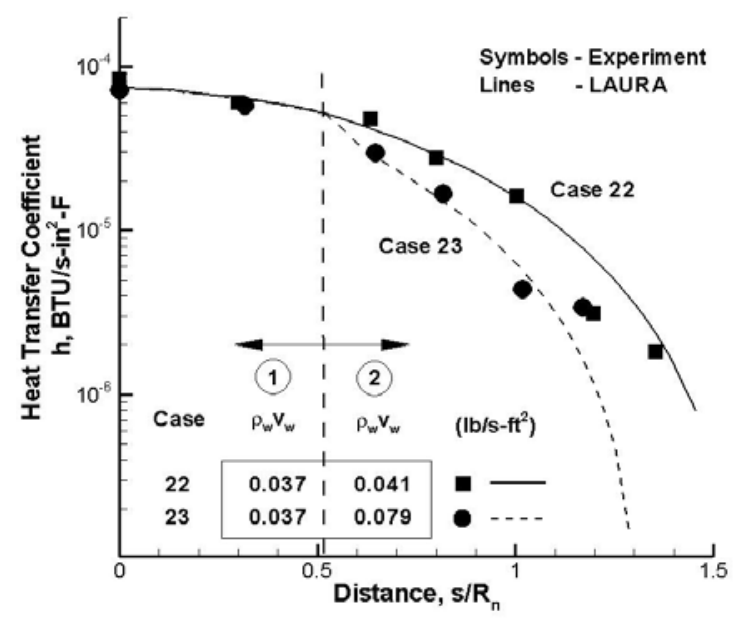

b) Blowing over the entire surface

Figure 5. Comparison of heat transfer coefficients on a sphere for different levels of mass transfer

The surface blowing in Fig. 5(a) corresponds to cases where there was zero mass transfer in the stagnation region (Region 1) prior to a surface distance of $s / R_{n}=0.52$. Downstream of that location, the mass transfer was set at 1times and 2-times the nominal rate which resulted in reduced surface heating over the sphere shoulder (Region 2) as shown in the figure. In Fig 5(b), a nominal blowing rate was introduced in the stagnation region while the downstream mass transfer rates were approximately the same as in Fig. 5(a). With blowing over the whole surface (Fig. 5b), the heating is lowered by $\sim 30 \%$ at the stagnation point with additional reduction over the shoulder depending on the blowing rate. In both figures, there is good agreement between the measured heating and the values computed with LAURA. Some discrepancy with the data is noted around $s / / R_{n}=1.2$ but the measurement appears spurious at that station.

The tests by Kaattari (Ref. 14) consisted of a hemisphere geometry and a blunted spherical segment representative of a capsule heatshield and so are of considerable interest in relation to CEV. Additional tests of a 
blunt conical body were reported in Ref. (14) but those data were not studied in this work. Figure 6 presents the experimental setup for the two models considered herein. Each model consisted of a permeable header in the shape of the hemisphere or spherical segment which was attached to a pressurized chamber in the body of the model. Mass flow rates through the permeable surface were calibrated during pretest based on the tunnel total pressure, surface pressure (predicted or measured), and flow conductance of the porous surface. A result of the calibration was that the mass flow varied along the surface with a lowering of injection rates in the stagnation region of the hemisphere model. The tabulated mass flow rates from the calibration and reported in Ref. 14 were matched exactly in the CFD calculations.

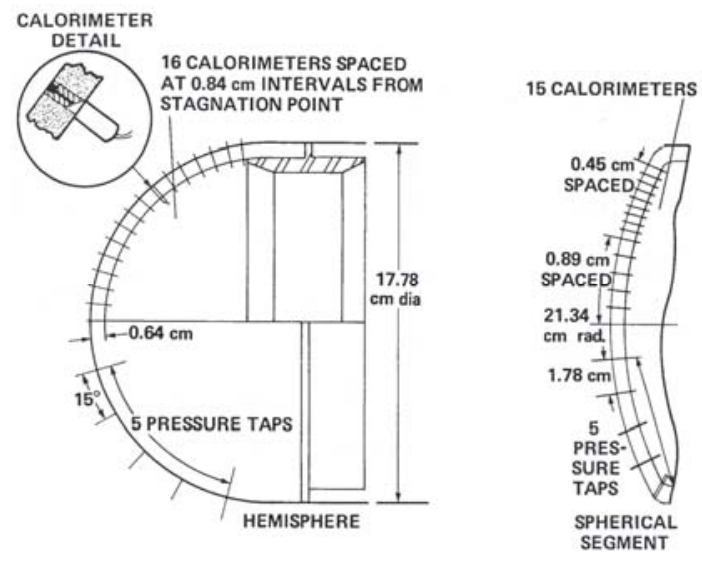

Figure 6. Blowing test model for hemisphere and spherical segment from Ref. 14

Predictions of the surface heat transfer using LAURA for the two shapes studied are compared to the measured heating rates at different levels of blowing in Figs. 7(a) and 7(b). Freestream conditions for both cases were at Mach 7.32 and a total pressure of $552 \mathrm{~N} / \mathrm{cm}^{2}$. At the lowest level of blowing, the prediction and measurement are in good agreement on the hemisphere but have increasing disparity in the stagnation region as the mass flow is increased. In the case of the spherical segment (Fig. 7b), the agreement between measurement and prediction is not good in the stagnation region but improves substantially in areas away from stagnation region for both levels of mass transfer. In these comparisons, the data measurements indicate that the reduction in heating at the stagnation point is substantially less sensitive to the mass transfer rate than was predicted by LAURA. The reason for disagreement between some of the data and the predictions is not completely understood, however, it was noted in Ref. 14, that the data from Feldhuhn (Ref. 13) suggests a similar level of insensitivity when the flow in the injection region was turbulent. Any such turbulent flow effects not modeled in the computations could explain the discrepancies. Overall, the comparisons between prediction and measurement were inconclusive for these data.

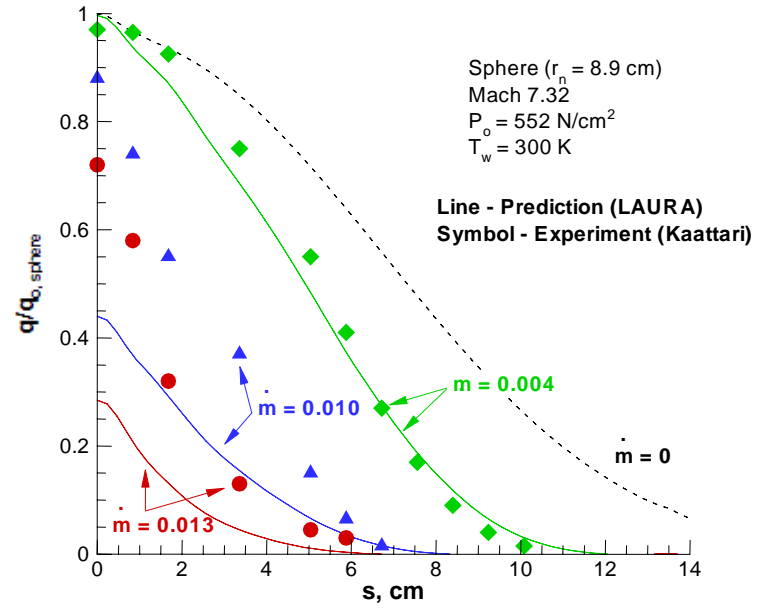

a) Hemisphere

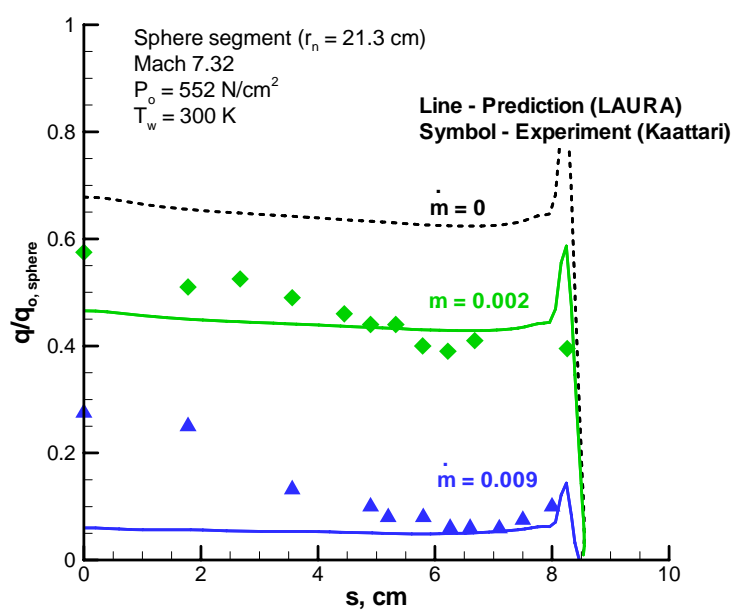

b) Spherical segment

Figure 7. Comparison of heat transfer ratio with mass transfer for data of Ref. 14 
The data published by Marvin and Akin (Ref. 15) offers different flow physics for validation of the blowing model in LAURA. In that experimental work, a 5-deg cone was fitted with a impermeable nosetip for the first 3.75 in of vehicle length followed by a porous conical section. Heat transfer measurements were made using air, argon, and helium injection along the cone surface although only air injection was considered in this work. The model was fitted with both sharp and blunt noses while freestream Reynolds number and injection rate were varied to investigate the effects of those parameters on boundary layer transition. Since the experiment was designed to promote transition on the conical flank, an adequate extent of laminar flow existed for most cases to make comparisons between measurement and LAURA predictions. The metallic experimental model had a uniformly porous surface that was calibrated so that mass flow rates along the vehicle length were accurately known. The calibration yielded a mass transfer distribution with $\sim 20 \%$ variation about the mean level. The reported distribution was input to LAURA as the surface boundary condition and calculations were done with four mean levels: $0 \%$, $0.04 \%, 0.08 \%$, and $0.12 \%$ of the freestream mass flow rate. The freestream Mach number was 7.4 , total temperature was $600 \mathrm{psi}$, and Reynolds number based on length was $4.7 \times 10^{6}$ for the cases computed. A comparison of the predicted heating rates with the experimental data is presented in Fig. 8 for the different blowing levels. In this figure, the heating rate is normalized by the experimental value of heating at the beginning of the porous cone section and plotted in the same coordinates and that were used in Ref. 15, however, some of the turbulent data that was measured is not included here for clarity. The experimental data in this figure clearly exhibits a transition to turbulent levels along the cone flank with the exact location dependent on the level of blowing. The transition is most forward at the highest levels of injection as would be expected. Excellent agreement between the measurement and computation was found for these data and served to further validate the boundary condition implementation in LAURA.

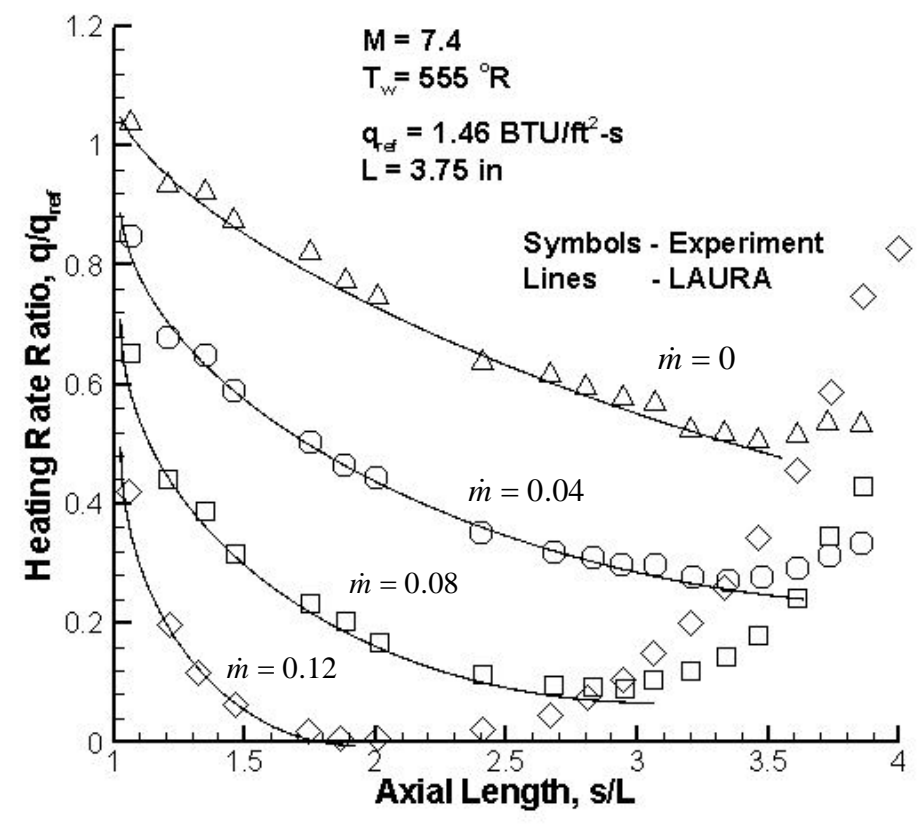

Figure 8. Heating rate ratio for sharp cone with air injection 


\section{Application}

Since the aim of implementing the blowing boundary condition into LAURA is to enable coupled ablation analysis it was of interest to demonstrate this capability for a test problem. Results have been published (Ref. 2) using a suite of codes to analyze the timedependent ablation of a 7-deg sphere-cone reentry vehicle (RV) with a 1.5 inch radius carbon nosetip. The simplified geometry, single carbon ablation element, and extensive published results offer a good benchmark for a demonstration problem. Kuntz, et.al. (Ref. 2) used a 2-dimensional in-depth material response code (COYOTE) in their work and allowed for shape change of the nosetip during the analysis. In the present work, the nose shape was allowed to vary in response to the ablation, however, a one-dimensional indepth response code (FIAT, Ref. 12) was employed and so the material response was expected to vary somewhat from previous results. Another difference in the present work in comparison to Ref. 2 was that a simple marching of the coupled problem was employed herein, whereas a predictor-corrector scheme was employed by the authors in Ref. 2. In their approach, the aerothermodynamic environment for each time step was computed by a CFD code and then corrected by iteration at each time step. It was reported (Ref. 2) that this approach was necessary for stability of the coupled analysis due to the high rate of ablation (and shape change) at later times along the RV ballistic trajectory. In the present work, the aerothermodynamic environment was computed at $\sim 1 \mathrm{~s}$ time steps and held fixed while the material response was analyzed over that period. The whole analyses was ended before the period of massive ablation was reached to avoid stability problems. The overall procedure employed for the coupled analysis is diagrammed in Fig. 9 where the various codes employed are named in the boxes and the arrows between boxes show the dataflow from code to code. This procedure was essentially the same as reported in other coupled analyses (Refs. 1,2). The ACE code (Ref. 18) in Fig. 9 was used to compute the equilibrium species at the surface once the pressure and temperature were known from the flowfield (LAURA) and material response (FIAT) codes. Shape change of the vehicle geometry was determined from the recession computed in FIAT and regridding for the next flowfield solution was done using a code (MVGRD) written for this test problem. Figure 10 presents the original surface and final ablated shape and associated flowfield grids after a total of ten time steps were analyzed following the methodology described.

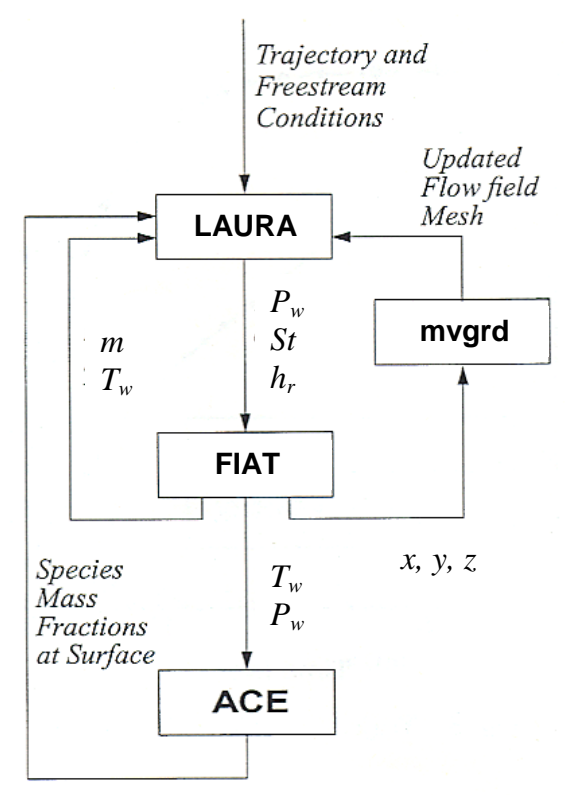

Figure 9. Coupling procedure for ablation anlaysis 


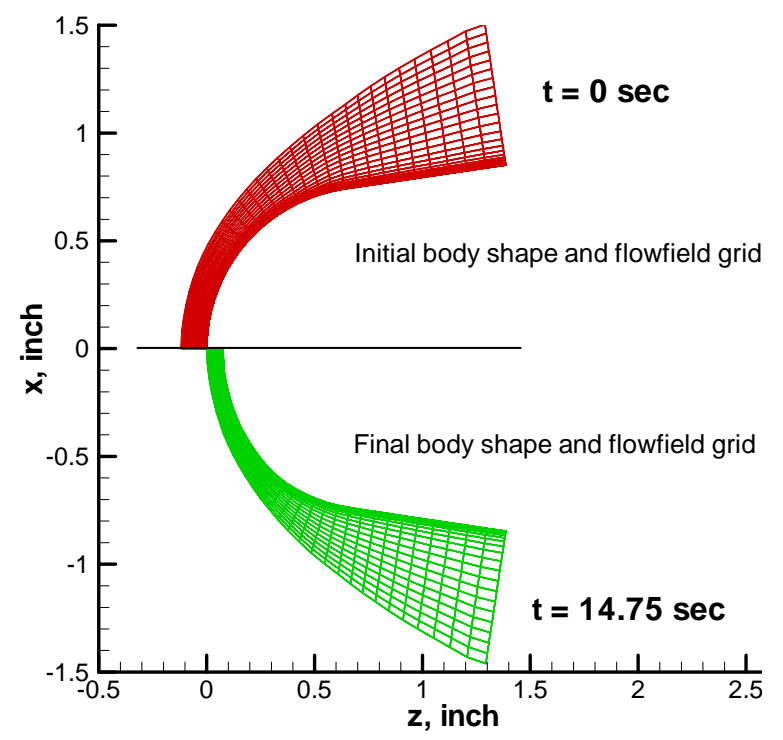

Figure 10. Initial and final (ablated) surface and flowfield grids

Figures 11(a) and (b) compares the predicted stagnation point recession and wall temperature in comparison to the results from Ref. 2. Good agreement with those published data was found thereby demonstrating that a coupled analysis capability using the LAURA code to provide the aerothermodynamic environment with ablation mass transfer at the surface.

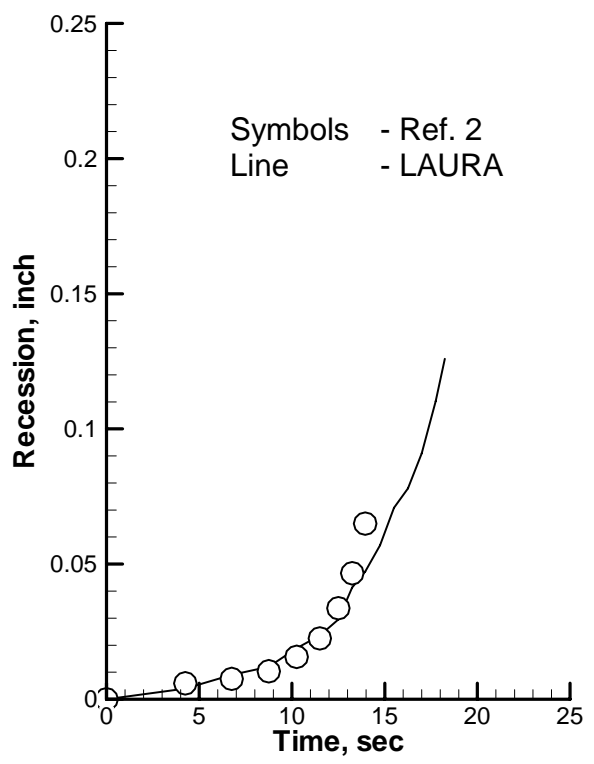

a) Surface recession

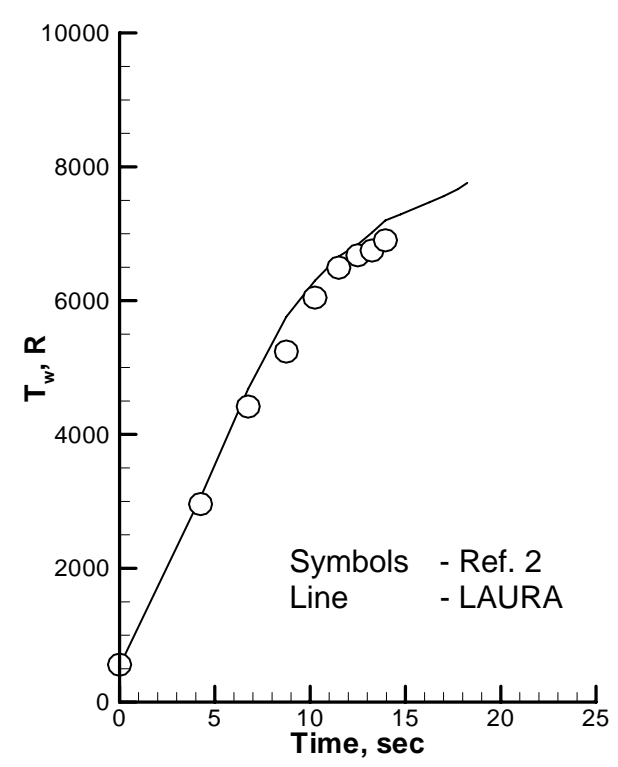

b) Surface temperature

Figure 11. Comparison of coupled ablation predictions with results of Ref. 2 


\section{Conclusion}

The implementation of a boundary condition with mass transfer (blowing) in the LAURA code proved to be a straightforward task and a range of mass transfer rates have been modeled in test cases with air being the primary injectant. Comparisons of the predicted velocity profiles with tabulated Blasius data showed good agreement as did a comparison of the reduction in Stanton number due to mass transfer from an analytic Couette flow solution. The capability to predict heat transfer in the presence of blowing was validated by comparisons with blunt nose and sharp cone data. Solutions with finite-rate chemistry in the flowfield and injection of carbon at the surface were also obtained in a coupled analysis of ablation and surface recession. Comparison of those results with previously published data for wall temperature and nosetip recession showed good agreement and demonstrated new capability. Computer calculations for the range of cases considered have shown that the CFD solutions behaved robustly and convergence was easily achieved. Computational performance with more complex surface chemistry (i.e. more and varied injectant species) remains to be measured but it was determined that the code and coupled ablation procedure is suitable for further applications.

\section{References}

1. Olynick, D., Chen, Y. K., and Taubet, M. E., “Aerothermodynamics of the Stardust Sample Return Capsule,”, Journal of Spacecraft and Rockets, Vol. 36, No. 3, pp. 442-462, 1999.

2. Kuntz, D.W., Hassan, B., and Potter, D.L., "Predictions of Ablating Hypersonic Vehicles Using an Iterative Coupled Fluid/Thermal Approach”, Journal of Thermophysics and Heat Transfer, Vol. 15, No. 2, pp 129-139.

3. Suzuki, K. and Kubota, H., "Chemical Nonequilibrium Ablation Analysis of Muses-C Super-Orbital Reentry Capsule,”, AIAA Paper 97-2481.

4. Gnoffo, P. A., “An Upwind-Biased, Point-Implicit Relaxation Algorithm for Viscous, Compressible Perfect-Gas Flows,” NASA TP 2953, 1990

5. Martinelli, S., Ruffin, S., McDaniel, R., Brown, J., Wright, M., and Hash, D., "Validation Process for Blowing and Transpiration-Cooling in DPLR,” AIAA Paper 2007-4255, June 2007.

6. Emmons, H.W. and Leigh, D., “Tabulation of the Blasius Function With Blowing and Suction,” Harvard Univ. Div. Applied Sci. Combustion Aerodynamics Lab. Iterim Tech. Report No. 9 (DDC Report No. AD27068), 1953.

7. Mickley, H.S., Ross, R.C., Squyers, A.L., and Stewart, W.E., "Heat, Mass, and Momentum Transfer for Flow over a Flate Plate with Blowing or Suction,” NACA Technical Note No. 3208, 1954.

8. Lees, L., “Convective Heat Transfer with Mass Addition and Chemical Reactions,” Combustion and Propulsion, Third AGARD Colloquium, pp. 451-498, Pergamon Press, New York, 1959.

9. $\quad$ Dorrance, W.H., Viscous Hypersonic Flow, McGraw-Hill, New York, 1962, pp. 58-59.

10. Kays, W.M. and Crawford, M.E., Convective Heat and Mass Transfer, 3rd edition, New York, McGraw-Hill, 1993, pp. 228-229,288-289.

11. Moyer, C. B., and Rindal, R. A., “An Analysis of the Coupled Chemically Reacting Boundary Layer and Charring Ablator, Part II: Finite Difference Solution for the In-Depth Response of Charring Materials Considering Surface Chemical and Energy Balances,” NASA CR-1061, June 1968, pp. 49-50.

12. Chen, Y. K. and Milos, F. S., “Ablation and Thermal Response Program for Spacecraft Heatshield Analysis,” Journal of Spacecraft and Rockets, Vol. 36, No. 3, pp. 475-483, 1999.

13. Feldhuhn, R. H., "Heat Transfer from a Turbulent Boundary Layer on a Porous Hemisphere," AIAA Paper 76-119, January 1976.

14. Kaattari, G., "Effects of Mass Addition on Blunt-Body Boundary-Layer Transition and Heat Transfer", NASA TP1139, 1978.

15. Marvin, J., Akin, C.M., "Combined Effects of Mass Addition and Nose Bluntness on Boundary-LayerTransition”, AIAA Journal, Vol. 8, No. 5, May 1970, pp. 857-863.

16. Libby, P.A. and Cresci, R. J., "Experimental Investigation of the Downstream Influence of Stagnation-Point Mass Transfer”, J. Aerospace Sci., Vol. 28. pp. 51-64, 1961.

17. Laganelli, A.L. and Martellucci, A., "Experimental Surface and Bopundary Layer Measurements in a Hypersonic Boundary Layer with Non-Uniform Blowing, AIAA Paper 74-699.

18. Anon., "User's Manual: Aerotherm Chemical Equilibrium Computer Program," Report UM-81-11/ATD, Acurex Corp., Mountain View, CA, Aug. 1981 